\title{
Community based universal neonatal hearing screening by health visitors using otoacoustic emissions
}

\author{
M Owen, M Webb, K Evans
}

\begin{abstract}
Objectives-To carry out a pilot study to test the feasibility of health visitors (HVs) performing neonatal otoacoustic emissions (OAE) hearing screening in the community using Echoport ILO288 and to evaluate its acceptability to parents and HVs.

Design-Prospective cohort study.

Setting-Local health centres and babies' homes in urban and rural settings in West Gloucestershire.

Participants-Twelve HVs, 683 babies, and their parents.
\end{abstract}

Main outcome measures-Coverage rate, age at testing, referral rate for formal audiology testing, and parental anxiety scores.

Results-Of the 683 babies registered with the study HVs, 99\% (675) were tested, with a median age at first test of 18 days. Parental consent for the study was refused for six of the eight not tested. Taking a unilateral pass as a screening pass (for comparison with other studies), 4\% (27I 675) failed the first OAE test, and $1.9 \%$ (13/675) failed a second OAE test performed by the HV within a further two weeks and were referred for formal audiology testing. One baby $(0.15 \%)$ was found to have a moderate sensorineural hearing loss on brain stem auditory evoked responses, giving a false positive rate of $1.7 \%$ (12/675). Some 18\% (120/675) were tested at home, of which $80 \%(96 / 120)$ were combined with another planned reason for $\mathrm{HV}$ contact. In all, $82 \%(555 / 675)$ of tests were carried out in health centre clinics, of which $47 \%$ (260/555) were combined purpose visits. Mean parental anxiety scores (possible range $0-5$ ) were $0.86,2.27$, and 3.45 before the first test, first retest, and audiology test respectively. The median time taken for one $\mathrm{HV}$ to complete testing was 12.2 minutes (range 3-65), compared with the 15 minutes currently allocated for two HVs to perform distraction testing. Based on the results of questionnaires, the test was very well received by parents and HVs alike.

Conclusion-HVs are able to perform OAE testing in the neonatal period at home and in local health centre clinics. They achieve high population coverage rates and low false positive rates. Universal neonatal hearing screening by HVs using OAE testing is feasible, well received, and could be less demanding of $\mathrm{HV}$ time than the current distraction testing. This model of universal neonatal hearing screening should be considered by the National Screening Committee.

(Arch Dis Child Fetal Neonatal Ed 2001;84:F157-F162)

Keywords: hearing; screening; health visitors; otoacoustic emissions

Permanent congenital hearing impairment has an incidence of 1.1 per 1000 live births. Early diagnosis is essential, to permit early treatment and rehabilitation, which are known to improve the outcome for deaf children in the development of language and communication skills. ${ }^{1}$ In most areas of the United Kingdom, the current screening procedure is the health visitor distraction test (HVDT), but this has the disadvantage that it cannot be performed until 6 months of age and it has a high false positive rate and relatively poor coverage. ${ }^{2}$

Electrophysiological methods of hearing screening have been widely used for both targeted and universal neonatal hearing screening (UNHS) in Europe and the United States. ${ }^{3-5}$

UNHS is currently being considered by the National Screening Committee. The precise method of administering the test to meet accepted screening standards, while also achieving optimal cost effectiveness, is a matter of current debate.

The most commonly used model to date has been a hospital based screen employing a team of dedicated screeners measuring otoacoustic emissions (OAE) in neonates on the maternity unit before discharge. This is backed up by a recall clinic for the babies missed by the initial screen. ${ }^{45}$ Such a model works well in areas served by single large maternity units. Potential problems occur for home deliveries and in areas covered by smaller maternity units. Difficulties may arise in attending a hospital recall clinic in more rural areas.

OAE testing is more likely to give false positive results in the first 24 hours of age. ${ }^{4-6}$ The ideal age for testing would therefore be beyond the first day of life, but this leads to difficulties in units where, as is increasingly common, babies are regularly discharged within the first 24 hours of birth. The current discharge rate within the first 24 hours at Gloucestershire Royal Hospital maternity unit is $28 \%$. In one North London maternity unit the rate is $48 \%$ (H Bantock, personal communication). 
Assuming that babies born outside the main maternity hospital (currently $2 \%$ at home and $20 \%$ in other maternity hospitals) and those discharged before 24 hours of age would not be covered by an initial inpatient screen at Gloucestershire Royal Hospital, the predicted population coverage of West Gloucestershire babies by this method alone would be around $50 \%$.

These considerations led us to consider a community based screen, testing children in a primary care setting in the first few weeks after birth. The technique of $\mathrm{OAE}$ testing is relatively easy to learn and machines can be preprogrammed with pass/fail criteria to reduce observer error. Specific qualifications in audiology are not essential for OAE screeners, ${ }^{4}{ }^{5}$ but testers need to have good baby handling skills and be able to communicate information sensitively and accurately to parents. Health visitors are well aware of the importance of early detection of hearing loss as part of their role in the continued surveillance of hearing and language development in preschool children to detect acquired and progressive hearing loss. ${ }^{2}$

Within a neonatal hearing screening programme using OAE, only babies with congenital hearing impairment at the cochlear level will be detected. A very small number of babies have defects of the auditory nerve, brain stem, or auditory cortex. These usually have had other risk factors for neurodevelopmental problems such as extreme prematurity, low birth weight, prolonged need for neonatal intensive care, perinatal asphyxia, convulsions, certain neonatal infections, abnormal cranial imaging, or critically high levels of bilirubin. This is a relatively small group (about $7 \%$ of total births at the Gloucestershire Royal), who routinely enter a full neurodevelopmental follow up programme. These infants are screened before discharge from the intensive care baby unit by automated brain stem responses, which allow testing of the normal hearing pathway to brain stem level. Cortical hearing loss is rare, but is checked for by behavioural responses at 1 and 2 years of corrected age as part of the neurodevelopmental follow up programme. These babies do not receive community based OAE screening.

A further concern, which applies to all neonatal screening programmes, is that of adverse psychological effects on parents caused by the screening process itself. This is particularly so if false positive or negative results are obtained, as reported by Hall et al in the case of Down's syndrome screening. Watkin ${ }^{8}$ studied anxiety in 288 mothers whose babies had received OAE screening in a hospital maternity unit. In general, the results indicated that excessive anxiety had not been caused, but there is relatively little published information on UNHS and we are not aware of any information relating to community based programmes. We therefore included measures of parental anxiety in this study.

\section{Methods}

Fourteen volunteer health visitors (HVs) from four rural and four city health centres took part in this study. Local ethics committee approval was obtained. All newborn babies registered with participating health centres were offered neonatal hearing screening by HVs using OAE during the trial period, January-December 1999. The only exceptions were babies at higher than normal risk of neurological hearing impairment who had been tested by automated brain stem responses before discharge from the neonatal unit.

HVs undertook a training programme which consisted of an introductory study day, followed by individual attendance at the targeted neonatal screening clinic at Gloucestershire Royal Hospital where practical training was given by a qualified audiologist. Final supervision and assessment of competence within the health centre setting was undertaken by an experienced tester. Back up support was available from the hospital audiology department, and all tests were stored on disc and reviewed later for quality control by a qualified person.

Transient evoked otoacoustic emissions were measured using ILO288 Echoport machines (Otodynamics, Hatfield, Herts, UK), preadjusted to settings matching the Wessex and Bath neonatal screening trials with automatic pass/fail criteria. ${ }^{5}$ Using the "Quickscreen" protocol, an automatic pass was registered if the overall response level was $28 \mathrm{~dB}$ or more, or the whole response correlation was $98 \%$ or more, or the signal to noise difference in any three wavebands reached a target level of $3 \mathrm{~dB}$ at 0.8 and $1.2 \mathrm{kHz}$ or $6 \mathrm{~dB}$ at $2.4,3.6$, and 4.0 $\mathrm{kHz}$. Two machines were available, and the HVs were responsible for transporting the machine between eight health centres.

District midwives gave information leaflets to the parents before transferring care of the newborns to the $\mathrm{HV}$ at the age of 10 days. Having obtained informed consent, the HV arranged an appointment between 10 and 24 days (correcting for prematurity). The HV was free to choose whether to perform the test at home or in a health centre clinic and whether the appointment should be solely for hearing testing or combined with another reason for contact.

Babies who passed the initial screening test were transferred directly into the continuing surveillance programme conducted by the $\mathrm{HVs}$, to continue through to school age.

Babies who remained too unsettled to be tested after reasonable measures had been taken on their first visit were recorded as "not tested" and given a further appointment.

According to the original protocol, babies who passed the initial OAE test unilaterally were deemed to have satisfied the screening criteria, conforming to other reported trials, but this policy was changed after the first 200 tests because it was found that parents were not satisfied if the second ear was not shown to be hearing normally. Subsequently, all bilateral and unilateral fails were retested by the $\mathrm{HV}$ within two weeks. Second fails were referred to the audiology department at Gloucestershire 
Royal Hospital within a further two weeks, where a third OAE test was carried out before proceeding to full brain stem auditory evoked responses (BSAERs). A paediatrician and ENT consultant were available on site for advice, and full facilities were available for continuing treatment and support of the child and family if permanent congenital hearing impairment was diagnosed.

DATA COLLECTION

Basic patient data and the outcome of testing were recorded on the child health computerised record system. Additional data collected were the age at testing, failures to attend, venue of testing, sole or combined visit, and time spent (a) travelling to home visits, (b) waiting for a baby to settle, and (c) performing the OAE test. Special difficulties such as external noise or an unsettled baby were noted. Similar data were collected for the first retest by the $\mathrm{HV}$, and the date and outcome of hospital referral were noted.

At the end of the whole screening procedure, parents were asked to complete a simple questionnaire, recording how anxious they had felt before the test (or retest) on a scale of 0 (not anxious) to 5 (very anxious indeed). They were also invited to give their views in free text on "What did you like about the way the test was done?" and "What was not so good about the way the test was done?" These data and comments were then analysed by a team of three clinical psychologists, who classified and coded the parental remarks, using standard techniques to ensure interobserver correlation. Further tests were applied six months later to parents of children who had failed the initial screening procedure (with matched controls) to examine long term effects of a screening failure. This research forms the basis of a separate study, which will be reported elsewhere.

In a final questionnaire, the views of participating HVs were sought on adequacy of training and support, experience of sharing the machines and views on optimum availability, appropriateness of neonatal OAE screening within the HV role, and as a service development for West Gloucestershire. Free text comments were also invited.

\section{Results}

Of the 683 neonates registered with the participating HVs on the child health computer record, only eight $(1.2 \%)$ were not tested (six for lack of parental consent and two for persistent non-attendance), leaving an overall coverage of $98.8 \%$. In total, $664(97.2 \%)$ babies were brought to the first confirmed appointment or the HV was notified of their inability to attend.

The HVs elected to carry out most of the tests $(82 \%)$ in a clinic setting in their local health centre. However, when the four urban and four rural practices were analysed separately, significantly more home tests were carried out in rural $(30 \%)$ than in urban settings $(8 \%)\left(\chi^{2}=37.7 ; \mathrm{p}<0.001 ;\right.$ table 1$)$.
Table 1 Number of tests carried out at home/clinic according to urban or rural location

\begin{tabular}{llll}
\hline Location & Tested in clinic & Tested at home & Total \\
\hline Rural & 217 & 91 & 308 \\
Urban & 338 & 29 & 367 \\
Total & 555 & 120 & 675 \\
\hline
\end{tabular}

Table 2 Sole purpose/multipurpose visits according to place of testing

\begin{tabular}{llll}
\hline Place of test & Multipurpose visit & Sole purpose visit & Total \\
\hline Home & 96 & 24 & 120 \\
Clinic & 260 & 295 & 555 \\
Total & 356 & 319 & 675 \\
\hline
\end{tabular}

Table 3 Time allocation

\begin{tabular}{lll}
\hline & Median (min) & Range (min) \\
\hline Settling time $(\mathrm{n}=675)$ & 2 & $0-45$ \\
Testing time $(\mathrm{n}=675)$ & 10.2 & $3-40$ \\
Total testing time $(\mathrm{n}=675)$ & 12.2 & $3-65$ \\
Travelling time $(\mathrm{n}=120)$ & 10 & $0-30$ \\
\hline
\end{tabular}

Overall $53 \%$ of OAE tests were performed in combination with other reasons for $\mathrm{HV}$ contact. Significantly more home tests $(80 \%)$ than clinic tests $(47 \%)$ were combined with another scheduled reason for contact (table 2; $\left.\chi^{2}=43.5 ; \mathrm{p}<0.001\right)$.

Table 3 gives the median times taken to carry out the test. Although there was wide variation, 282 babies $(42 \%)$ required no settling time. In cases where the baby was unsettled, the HV was able to use the time profitably while the parent comforted the baby. The total testing time (median 12.2 minutes) refers to the sum of the settling time plus the actual testing time. This compares with an allocated booking time of 15 minutes for two HVs to perform one HVDT.

The median age at first testing was 18 days, range 7-40 days (plus two babies aged $>40$ days who moved into the area after birth). The median age of testing at the hospital audiology clinic (after two OAE test failures with the HV) was 38 days (range 27-120 days). Except for the child seen at 120 days (parent unable to bring sooner), all completed full audiological assessment well before the age of 3 months as recommended by the American Academy of Pediatrics. $^{9}$

OUTCOMES

Figure 1 shows the outcomes of testing. For ease of comparison with other reported studies, unilateral passes are counted as screening passes. However, there were 20 unilateral failures on the first test, and, of these, five received no further testing (part of the first 200 cohort), 10 passed at a second OAE test, and five failed the second OAE test and were referred to audiology where all five passed BSAER testing.

Of the total 675 babies tested, the first test pass rate was $92.7 \%$ (bilateral) and $95.7 \%$ (unilateral). After the second test, the pass rate was $96.3 \%$ (bilateral) and $97.7 \%$ (unilateral). Only one $(0.15 \%)$ child was found to have a moderate bilateral hearing loss on BSAER. 


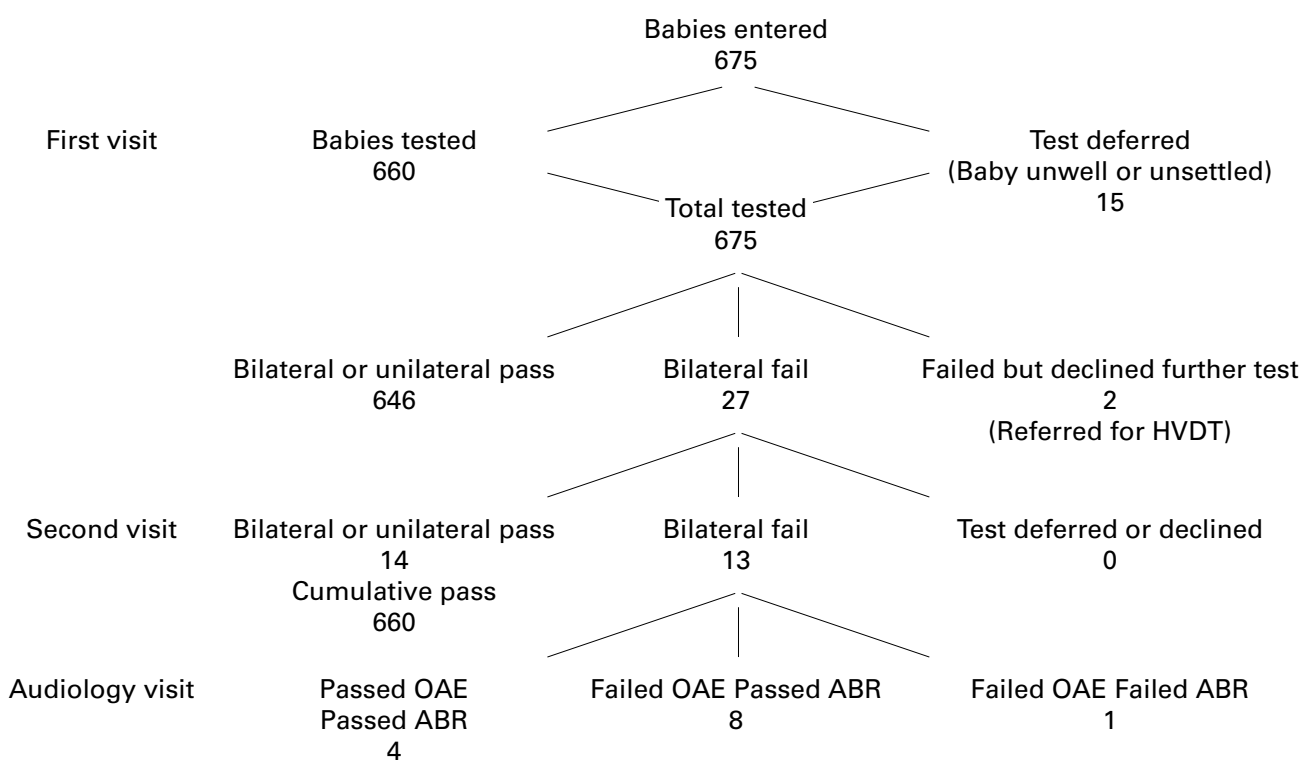

Figure 1 Test outcomes. Unilateral passes are counted as screening passes. HVDT, Health visitor distraction test; OAE, otoacoustic emissions test; ABR, automated brain stem responses test.

PARENTAL ANXIETY

Figure 2 summarises the results of parental anxiety scores. In total, 474 questionnaires were returned. Scores (on a scale from $0=$ not anxious to $5=$ very anxious indeed) were divided into three groups according to whether the baby was tested once $(n=439)$, twice $(n=$ 13), or three times $(n=22)$. These included both bilateral and unilateral fails and some babies who were tested according to the protocol but after the main study had ceased to accrue cases for analysis.

Before the first test, there was no significant difference in anxiety levels between the three groups. Mean anxiety score was $0.86 ; 54 \%$ of respondents reported an anxiety score of level 0 , and $0.7 \%$ of level 5 . Before the second test, the mean anxiety score was $2.27 ; 16 \%$ of respondents reported anxiety score of level 0 , and $3.3 \%$ of level 5 . Before the third test (hospital visit for full diagnostic programme), the mean anxiety score was 3.45 with no anxiety levels of 0 or 1 and $21 \%$ reporting level 5 .

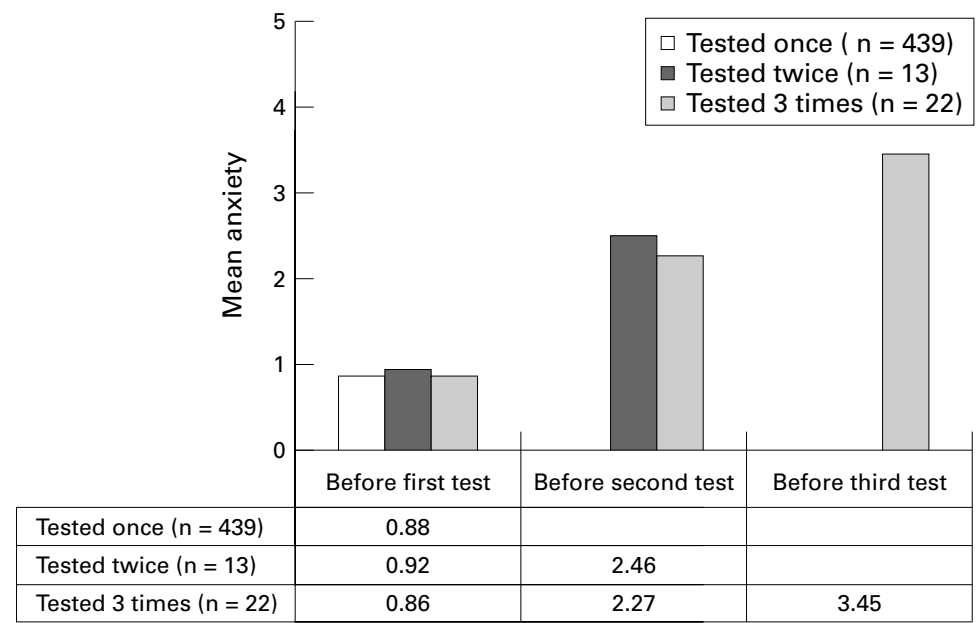

Figure 2 Mean parental anxiety scores before first, second, and third test. The total number of respondents was 474. The anxiety scale ranges from "not at all anxious" (0) to "very anxious indeed" (5).
Despite experiencing anxiety in some cases, when asked to comment on their babies' hearing tests, nearly all mothers were very positive, with very few making any criticisms. They liked the speed $(n=225)$ and simplicity $(n=112)$ of the procedure, that it did not upset or cause any discomfort to their babies $(n=233)$, and that the test could be carried out at such a young age $(n=48)$. The few criticisms mainly concerned the probe falling out $(n=27)$ or the need for silence $(n=42)$.

Parental attitude to unilateral screening passes, shown both by direct comments to the $\mathrm{HV}$ and in three free comments on the first 200 questionnaires to be returned (representing $100 \%$ of unilateral fails in that cohort), was that they were anxious about the status of the ear that had not recorded a pass, even though their baby had been shown to have normal hearing in one ear. All requested a further test, and from that point retests were routinely offered to all unilateral fails.

HV QUESTIONNAIRE

All $14 \mathrm{HVs}$ returned questionnaires. All felt that training and support had been adequate and that neonatal hearing screening using OAE was an appropriate role for them, and one that they would like to see implemented throughout the whole of West Gloucestershire. Eleven felt that they could manage with the machine being available for half day a week, whereas three felt that they would need it for a whole day a week. Eight had experienced problems with sharing machines, especially in rural areas where distances between health centres were large. All experienced difficulty in transporting the Echoport machine because of its weight and the security hazard of its separate lap top computer, but felt that these difficulties could be overcome by using the newer hand held screeners.

Neonatal OAE testing was felt to be a natural extension of the HV role in the newborn 
period and all HVs expressed a strong preference for the OAE test over HVDT. They appreciated the enhancement of their professional image and the basis created for their future responsibility in detecting late onset and progressive hearing loss, once the HVDT is replaced by neonatal screening.

\section{Discussion}

We have shown that it is not only feasible for $\mathrm{HVs}$ to perform neonatal hearing screening in the community, but that it is possible to achieve very high coverage rates and low false positive rates. The procedure is highly acceptable to both HVs and patients and has a potential for time (and therefore cost) savings.

Currently there is pressure from the medical professions, organisations representing deaf children, and the general public to replace the present HVDT at 6 to 7 months of age by UNHS. The future introduction of UNHS is being considered as a priority by the National Screening Committee. Debate centres around which method of screening should be used (OAE $v$ automated brain stem audiology $(\mathrm{AABR})$ ) and where the test should be conducted (hospital $v$ community) in order to meet effective screening standards. ${ }^{9-11}$

The Wessex trial ${ }^{5}$, using a cross over study based on four maternity units, showed that hospital based UNHS compares favourably with HVDT at 6 to 9 months. Most published studies from the United States and Europe used inpatient UNHS screening programmes involving $\mathrm{OAE}$ or $\mathrm{AABR}$ testing. Published research on community based screening is scarce. A Dutch study describes OAE testing by midwives (B Van Zanten. Community based otoacoustic emissions screening in the Netherlands. Baby BSA British Society of Audiology Annual Conference, Winchester, 1996), as has Williamson in Bath ( $\mathrm{T}$ Williamson. Neonatal hearing screening by community midwives: a pilot study. Personal communication). Bantock and Croxson ${ }^{11}$ describe a programme using trained screeners operating in a community clinic in Islington, London. In Flanders, UNHS has been carried out since 1997 by HVs using AABR. The programme has been extremely successful, with only $0.17 \%$ of the first 110000 babies tested being referred for hospital audiology testing after two failed AABRs with the HV. The same advantages for early discharges and home deliveries applied as with the Gloucestershire study, and the Flemish HVs experienced similar enhancement of their role in the newborn period and in further surveillance of children who passed the initial hearing screen. However, the cost of disposable electrodes and ear pieces used in this method is in excess of $£ 5.00$ per baby, although some of this may be offset by the lower rate of retests and referrals (L Stappaerts. Health visitor based AABR programme in Belgium. The 13th Annual Workshop on Hearing Screening in Children, Nottingham, March 2000).

Our pilot study in West Gloucestershire is population based, the denominator being the total number of children allocated to the care of the participating HVs. As every neonate in this county is allocated to an HV, the coverage figures in this study should be achievable for all births in West Gloucestershire if all HVs participated in the screening programme.

Our coverage rate of $99 \%$ compares extremely favourably with other published figures of $91 \%{ }^{5}$ and $92 \% .{ }^{4}$ Both these figures relate to babies born in the district general hospital, therefore babies born in peripheral maternity units or at home are excluded. Coverage in the Wessex trial would fall to an average of $86 \%$ across the four participating hospitals (range $79-91 \%$ ) if figures were extrapolated to include all babies in the community. ${ }^{5}$ Although the introduction of a weekly recall clinic improved coverage by $4-5 \%$, this is still well short of our $99 \%$.

We used standard OAE equipment and protocols and did not attempt to assess the sensitivity or specificity of the method, as this has been shown elsewhere. ${ }^{35}$ We intended to duplicate the testing criteria of the Wessex and Bath studies, ${ }^{5}$ but after analysis of the first 200 parental questionnaires, we realised that, by accepting a unilateral pass, we were raising parental anxiety about the other ear. From that point onwards, the protocol was altered to require a pass in both ears.

It is well documented that OAE testing has a high false positive rate (up to $15.6 \%$ ) in the first 24 hours of life, falling to about $4 \%$ by 72 hours. ${ }^{56}$ Although some of this is related to middle ear effusion and debris in the external ear canal, it may also be related to neurological immaturity. ${ }^{612} 13$ The median age of testing in our study was 18 days, thereby eliminating the early neonatal problems while still allowing us to complete the full programme of screening and definitive testing well before the age of 3 months as recommended in 1999 by the American Academy of Pediatrics. ${ }^{9}$

The first test pass rate in our study was 92.7\% (bilateral) and 95.7\% (unilateral), rising to $96.3 \%$ and $97.7 \%$ respectively after the second test. The unilateral or bilateral screening pass rate of $97.7 \%$ in this study is comparable with that of the Wessex study of $98.4 \%$.

Of the 13 babies referred by the HVs to the audiology department for bilateral OAE test failures on two occasions, nine failed a third OAE test in the department, although all but one subsequently went on to have normal BSAER. This suggests that, in most cases, there was sustained inhibition of the OAE response by factors such as fluid, wax, or debris in the external or middle ear.

Parental anxiety is an important consideration in any neonatal screening programme, but few published studies have examined this aspect of UNHS. ${ }^{8}$ We attempted to allay anxiety as much as possible by providing information sheets and by personal discussion between the HV and parent. Anxiety scores were universally low before the first test, but not surprisingly rose with the need for each successive retest.

Overall the screen was extremely well received by parents, but further information on parental anxiety is needed. 
The HVs' pattern of work was closely studied to examine how UNHS would fit into the wider $\mathrm{HV}$ role in the newborn period. They were given freedom to choose whether to test babies at home or in a health centre clinic and whether or not to combine it with another scheduled contact. It is interesting to note that $30 \%$ of tests in rural areas were performed at home, compared with only $8 \%$ in urban areas (table 1). This is likely to represent a considerable attraction to some parents in rural areas where public transport is poor. This convenience for the patient is also at little extra cost to the service, as $80 \%$ of home tests were combined with another scheduled reason for visiting the home (table 2).

The advent of the new hand held OAE screening devices such as Echocheck or Echosensor (Otodynamics) may further facilitate community based screening provided that their sensitivity and specificity are comparable with those of standard equipment. Recent reports such as that of Reuter $e t a l^{6}$ are very encouraging, suggesting that both methods have a sensitivity of $100 \%$, with the specificity of ILO88 being $96.1 \%$ and that of Echosensor $93.3 \%$ on the initial test. We are therefore hopeful that hand held screening devices will be validated as appropriate equipment for UNHS.

By comparing the time taken to perform OAE testing in this study with that currently allocated to HVDT, it is possible to obtain an approximate comparison of manpower costs. We currently allocate 15 minutes for two HVs to perform an HVDT, amounting to 30 minutes of HV time per test. Our median total time required per OAE test (settling time + testing time) amounts to 12.2 minutes. Appointment intervals of 15 minutes were found to be adequate for OAE testing, but only one $\mathrm{HV}$ was required, thus halving the $\mathrm{HV}$ time allocation for each child's initial hearing test. The budget for HVDT could therefore be transferred directly to UNHS, with cost savings being redeployed to other $\mathrm{HV}$ activities such as a continued surveillance programme for detection of late onset and conductive hearing losses. Additional funding requirements include the cost of disposable rubber ear pieces at $£ 30.00$ per 100 , maintenance costs of the equipment, and the provision of quality control measures.

In calculating set up costs, we would assume that the equipment we used (Echoport ILO288; cost $£ 5550$ per machine) would be replaced by a cheaper and more convenient hand held screener-for example, Echocheck; cost $£ 1950$ per machine. There are 52 health centres in West Gloucestershire, serving a population of 339000 persons. A pool of 11 Echocheck machines would allow each practice the use of a machine for a whole day a week at a set up cost of $£ 21450$.
This study was designed to test the feasibility of UNHS by OAE testing carried out by HVs in the community. We acknowledge that numbers of participants are relatively small, and that the high coverage rates may not be reproducible outside the research setting, but the findings are sufficiently robust to merit serious consideration within the current debate over UNHS.

We feel that UNHS programmes based on this model would be applicable to many health districts in the United Kingdom. There is urgent need for further studies to compare hospital based and community based programmes in different settings and to establish the optimum testing procedure in the light of recent developments in the technology of neonatal hearing screening.

We are grateful to Mrs Ann Marshall, Senior Nurse, Health Visitor, Severn NHS Trust, and the participating health visitors, Cilla Couchman, Maggie Cocks, Jo Clements, Rachael Liley, Jennifer Berridge, June Reeves, Jo King, Karen Barnes, Patricia Harbottle, Sharon Stones, Lin Thomas, Laura Evans, Jane Tarbottle, Sharon Stones, Lin Thomas, Laura Evans, Jane
Toomer, and Sheena Hurn. Lindsay Kimm assisted with the training programme, and Christine Newcombe and Hilary Cant training programme, and Christine Newcombe and Hilary Cant
provided technical assistance in audiology. Chris Foy provided provided technical assistance in audiology. Chris Foy provided with analysis of the parental anxiety data.

Contributor

$\mathrm{M} \mathrm{O}$ initiated the research, developed the protocol, trained and supervised the HVs, wrote the information sheets, was responsible for the overall management of the project, data collection, and analysis, and participated in writing the paper. $\mathrm{M} \mathrm{W}$ and $\mathrm{KE}$ assisted in planning the research and in writing the paper. $\mathrm{M} \mathrm{W}$ is guarantor.

This research was supported by a grant from Children Nationwide.

1 Yoshinaga-Itano C, Sedley A, Coulter DK, et al. Language of early- and late-identified children with hearing loss. Paediatrics 1998;102:1161-71

2 Davis A, Bamford J, Wilson I, et al. A critical review of the role of neonatal screening in the detection of congenital hearing impairment. Health Technol Assess 1997;1:10.

3 White KR, Maxon AB. Universal screening for infant hearing impairment: simple, beneficial and presently justified. Int $\mathcal{f}$ Pediatr Otorhinolaryngol 1995;32:201-11.

4 Watkin P. Neonatal otoacoustic emission screening and the identification of deafness. Arch Dis Child Fetal Neonatal Ed 1996;74:F16-25.

5 Kennedy CR. Controlled trial of universal neonatal hearing screening for early identification of permanent congenital screening for early identification of permanent
hearing impairment. Lancet 1998;352:1957-64.

6 Reuter G, Bordgen F, Dressler F, et al. Neugeborenenhorscreening mit dem automatisierten Messgerat Echosensor fur otoakustische Emissionen. HNO 1998;46:93241.

7 Hall S, Bobrow M, Marteau T. Psychological consequences of false negative results on prenatal screening for Down's syndrome: retrospective interview study. $B M \mathcal{F}$ 2000;320:407-12.

8 Watkin P. Maternal anxiety and attitudes to universal neonatal hearing screening. Br f Audiol 1998;32:27-37.

9 American Academy of Pediatrics. Joint taskforce on newborn and infant hearing loss: detection and intervention. Paediatrics 1999;103:527-30.

10 European Consensus on Neonatal Hearing Screening. Acta Paediatr 1999;88:107-8.

11 Bantock $\mathrm{H}$, Croxson S. Universal hearing screening using transient otoacoustic emissions in a community health clinic. Arch Dis Child 1998;78:249-53.

12 El-Rafaie A, Parker D, Bamford J. Oto-acoustic emissions versus $A B R$ screening: the effect of external and middle ear abnormalities in a group of SCBU neonates. $B r f$ Audiol 1996;30:3-8.

13 Thornton A, Kimm L, Brocaar M, et al. External and middle ear factors affecting evoked otoacoustic emissions in neonates. Br F Audiol 1993;27:319-27. 